\title{
Kinetic equation of linear fractional stable motion and applications to modeling the scaling of intermittent bursts
}

\author{
N. W. Watkins, ${ }^{1, *}$ D. Credgington, ${ }^{1, \dagger}$ R. Sanchez, ${ }^{2}$ S. J. Rosenberg, ${ }^{1}$ and S. C. Chapman ${ }^{3, *}$ \\ ${ }^{1}$ Physical Sciences Division, British Antarctic Survey (NERC), Cambridge CB3 OET, United Kingdom \\ ${ }^{2}$ Fusion Energy Division, Oak Ridge National Laboratory, Oak Ridge, Tennessee 37830, USA \\ ${ }^{3}$ Centre for Fusion, Space and Astrophysics, University of Warwick, Coventry CV4 7AL, United Kingdom
}

(Received 3 July 2008; revised manuscript received 11 February 2009; published 16 April 2009)

\begin{abstract}
Lévy flights and fractional Brownian motion have become exemplars of the heavy-tailed jumps and longranged memory widely seen in physics. Natural time series frequently combine both effects, and linear fractional stable motion (lfsm) is a model process of this type, combining $\alpha$-stable jumps with a memory kernel. In contrast complex physical spatiotemporal diffusion processes where both the above effects compete have for many years been modeled using the fully fractional kinetic equation for the continuous-time random walk (CTRW), with power laws in the probability density functions of both jump size and waiting time. We derive the analogous kinetic equation for $1 \mathrm{fsm}$ and show that it has a diffusion coefficient with a power law in time rather than having a fractional time derivative like the CTRW. We discuss some preliminary results on the scaling of burst "sizes" and "durations" in lfsm time series, with applications to modeling existing observations in space physics and elsewhere.
\end{abstract}

DOI: 10.1103/PhysRevE.79.041124

PACS number(s): 05.40.-a, 89.75.Da

\section{INTRODUCTION}

Fractional kinetics is finding increasingly wide application to physics, chemistry, biology, and interdisciplinary complexity science [1-6]. One reason for this is the link between "strange" kinetics and observed non-Brownian anomalous diffusion, motivating the use of fractional dynamical models of transport processes, including those based on fractional calculus. Scalas et al. [5] gave numerous applications; we will just note space plasmas [7], magnetically confined laboratory plasmas [8-11], fluid turbulence [4], and the travels of dollar bills [12].

Equally widespread in its application, and evolving in parallel with the theory of anomalous diffusion, is the theory of anomalous time series. The corresponding models, particularly in the mathematics and statistics literature, have often been based on stable self-similar processes [13-15]. Stability here means the property whereby the shape of a probability density function (pdf) remains unchanged under convolution to within a rescaling (cf. Chaps. 3 and 4 of [16]). It is an attractive feature in modeling, particularly when one anticipates that a signal represents a sum of random processes. In particular stable self-similar processes, a development in the wider field of stochastic processes $[17,18]$, can model two effects which are often seen in real data records. The firstMandelbrot's "Noah" effect [19]—describes non-Gaussian "heavy-tailed" pdfs, while the second-his "Joseph" effect [20]-manifests itself as long-ranged temporal memory. The many applications have included hydrology [21], finance [19], magnetospheric activity as measured by the auroral indices [22,23], in situ solar wind quantities [23], and solar flares [24].

\footnotetext{
*Also at the Kavli Institute for Theoretical Physics, Santa Barbara, California, USA.

†Present address: London Centre for Nanotechnology, University College London, London, UK.
}

The existence of two rich parallel, but intersecting literatures means that it is not yet completely known which techniques from one will apply to a given problem in the other. It is, for example, not always clear a priori what type of kinetic equations will apply in a given context. The right class of kinetic equation for reversible microphysical transport need not also be the right one for an evolving time series taken from a macroscopic variable. The problem of model choice is an important and timely one, in both physics and more general complexity research. Because different models can predict subtly different observable scaling behaviors, distinguishing them may require measuring several exponents, as any individual exponent may be identical across several models, a point emphasized by Lutz [25].

It is now increasingly recognized that much natural data are of the type that Brockmann et al. [12] dubbed "ambivalent." In such systems heavy-tailed jumps and long-ranged temporal memory compete to determine whether transport is effectively superdiffusive or subdiffusive. The ambivalent process (ap) they used for illustration, and fitted to data, was the well-studied fully fractional continuous-time random walk (CTRW) [3] which incorporates both effects via fractional orders of the spatial and temporal derivatives in its kinetic equation. Zaslavsky et al. [26,27] also advocated use of the same process in space physics for modeling the auroral index time series. They explicitly contested [27] the applicability of a time-series model [23] based on a self-similar stable process-linear fractional stable motion (lfsm) $[13,14]$ - in this role. We note that, rather than being purely a mathematical abstraction, lfsm has been linked to physics via the propagation of activity fronts in extremal models [28]. A comparison of these two approaches, leading to better understanding of their structure and their similarities and differences, thus seems to us to be highly topical. It will be the first of two main topics of this paper. Although we are fully aware that the kinetic equation we obtain on its own cannot fully specify a non-Markovian process and, importantly, will 
TABLE I. Kinetic equations for the main classes of process used to study anomalous time series and transport beyond the BachelierWiener-Brownian paradigm. Ordinary Lévy motion (oLm) parametrized by a stability exponent $\alpha$ relaxes the finite-variance assumption of the central limit theorem. The fractional time process (ftp) and the ap, i.e., the fully fractional continuous-time random walk, add a fractional derivative of order $\alpha H$ to the kinetic equations for Bachelier-Wiener Brownian motion (BWBm) and oLm, respectively. A different way of introducing temporal memory effects is via stable self-similar processes with memory kernels, fractional Brownian motion (fBm), and lfsm, respectively. Although not fully specified by them, the stable processes nonetheless have kinetic equations with time-dependent diffusion coefficients. The kinetic equation for lfsm is highlighted. In the self-similar stable processes the self-similarity parameter $H$ depends on a memory exponent $d$ and on the stability exponent $\alpha$ via $H=1 / \alpha+d$, and in the CTRW case, by contrast, on the standard memory exponent, $d^{\prime}=\alpha H$. In all cases there is a coefficient $D$ with appropriate dimensionality on the left-hand side which we have set to 1 . In the BWBm case this is simply the familiar diffusion coefficient.

\begin{tabular}{|c|c|c|c|}
\hline & & Stable process & CTRW \\
\hline$\alpha$ & $H$ & $H=1 / \alpha+d$ & $d^{\prime}=\alpha H$ \\
\hline \multirow[t]{2}{*}{$\alpha=2$} & $H=1 / 2$ & BWBm & \\
\hline & & $\nabla^{2} p=\partial_{t} p$ & \\
\hline \multirow[t]{2}{*}{$0<\alpha \leq 2$} & $H=1 / \alpha$ & $\mathrm{oLm}$ & \\
\hline & & $\nabla^{\alpha} p=\partial_{t} p$ & \\
\hline \multirow[t]{2}{*}{$\alpha=2$} & $0 \leq H \leq 1$ & $\mathrm{fBm}$ & ftp \\
\hline & & $2 H t^{2 H-1} \nabla^{2} p=\partial_{t} p$ & $\nabla^{2} P=\partial_{t}^{\alpha H} p$ \\
\hline \multirow[t]{2}{*}{$0<\alpha \leq 2$} & $0 \leq H \leq 1$ & Ifsm & ap \\
\hline & & $\alpha \mathbf{H t}^{\alpha \mathrm{H}-1} \nabla^{\alpha} \mathbf{p}=\partial_{\mathbf{t}} \mathbf{p}$ & $\nabla^{\alpha} p=\partial_{t}^{\alpha H} p$ \\
\hline
\end{tabular}

not be unique to lfsm, we nonetheless believe that our comparison of the kinetic equations for the two paradigms is of value, particularly as a source of physical insight (see also Secs. 1 and 2 of [29]).

To make the comparison we first briefly recap (Table I) the main kinetic equations corresponding to the modeling of time series by stable processes and of anomalous diffusion by the CTRW. In particular we highlight (following Lutz [25]) the difference between $\mathrm{fBm}$ and the ftp, which has sometimes led to confusion, at least in the physics and complexity literature (e.g., [23]). We illustrate the potential value of this comparison with reference to a surprising gap in the physics literature, the absence of a kinetic equation corresponding to lfsm, analogous to the one given for fBm by Wang and Lung [30]. We give a simple derivation by direct differentiation using the characteristic function. The kinetic equation can be obtained by methods as diverse as a transformation $t^{\alpha H}$ of the time variable in the space fractional diffusion equation, and a path integral [31].

Our second main topic is the potential relevance of lfsm to physics as a toy model for "calibrating" diagnostics of intermittency (cf. [32]). As a frequent attribute of nonequilibrium and nonlinear systems, intermittency has been a particular stimulus to physicists and time-series modelers [33]. In particular the paradigm of self-organized criticality (SOC)
[33] has been one framework for this, embodying the hypothesis of avalanches of activity in nonequilibrium complex systems. We investigate the scaling of intermittent bursts in lfsm, using the burst size and duration measures which have very often been used as direct diagnostics of SOC. Such measures have been previously studied on, among many others, magnetospheric and solar wind time series [34] as well as, for example, magnetohydrodynamic (MHD) simulations of turbulence [35-37]. We follow several earlier conjectures [22,34,37-39], and build on the heuristic scaling argument given by Kearney and Majumdar [40], to suggest that lfsm could indeed be one candidate model for observed power laws in such bursts. We test our arguments with numerics using the algorithm of Stoev and Taqqu [41]. We confirm the earlier results of Carbone et al. [38] and the more recent work of Rypdal and Rypdal [42]. These papers considered just the $\alpha=2$, fBm case, using a running average threshold and a fixed threshold, respectively. The scaling arguments of Carbone et al. [38] were independent of the type of threshold and with hindsight should also be expected to hold for the constant threshold case that we study.

We find numerically, however, that for lfsm our simple scaling argument, while giving a good approximation to the dependence of the burst exponents on the self-similarity exponent $H$ as $\alpha$ is initially reduced from 2, eventually becomes much less accurate as $\alpha$ reaches 1 . We conclude by offering some suggestions as to the reasons for this, and describing future work.

\section{KINETIC EQUATION OF lfsm}

\section{A. Limit theorems and stochastic processes}

In Table I we collect the kinetic equations for the pdf $p$ $=p(x, t)$ of some processes which have been proposed in the various literatures on time-series analysis and anomalous diffusion. For clarity we concentrate on the simplest examples from the family of stable processes and from the CTRW. In the table the (statistical) self-similarity exponent $H$ is defined using dilation in time where $\Delta t$ goes to $\lambda \Delta t$,

$$
x(\lambda \Delta t)=\lambda^{H} x(\Delta t),
$$

and the equality is in distributions.

The fourth row of Table I corresponds to BWBm and the fifth row to the familiar diffusion equation where we have abbreviated $\partial / \partial t$ to $\partial_{t}$. BWBm is of course a manifestation of the central limit theorem (CLT) $[43,44]$. The solution $p(x, t)$ is of Gaussian form with width spreading as $t^{1 / 2}$ and its characteristic function is also a Gaussian in $k\left[\sim \exp \left(-|k|^{2} t\right)\right]$ with stability exponent $\alpha=2$.

\section{B. Anomalous diffusion and the extended central limit theorem}

Similarly, the fifth row of Table I corresponds to relaxing the assumption of finite variance, by allowing a stability exponent $0<\alpha<2$. The corresponding pdfs $p_{\alpha}(x, t)$ are the $\alpha$-stable class, with power-law tails decaying as $x^{-(\alpha+1)}$. Following Mandelbrot we refer to these as "Lévy flights" or ordinary Lévy motion. 
The corresponding kinetic equation

$$
\frac{\partial p_{\alpha}(x, t)}{\partial t}=\frac{\partial^{\alpha} p_{\alpha}(x, t)}{\partial|x|^{\alpha}}
$$

has a symmetric Riesz fractional derivative in space, $\partial^{\alpha} / \partial|x|^{\alpha}$, which in Table I is given as three dimensional and abbreviated to $\nabla^{\alpha}$. The Riesz derivative is a pseudodifferential operator with symbol $-|k|^{\alpha}$ and $p_{\alpha}(x, t)$ has characteristic function $\hat{p}_{\alpha}(k, t)=\exp \left(-|k|^{\alpha} t\right)$. Unlike the cases we now go on to discuss, the kinetic equation for oLm is still unambiguously Markovian and an expression of the extended CLT. Due to infinite divisibility, in this specific case the pdf $p_{\alpha}(x, t)$ alone is enough to uniquely characterize the stochastic process, which we will call $Z_{\alpha}(t)$.

\section{Relaxing independence through temporal memory:} Fractional Brownian motion versus the fractional time process

The seventh row of Table I describes the case when the independent and identically distributed (iid) assumption is relaxed, rather than the finite-variance one. This case is more subtle than the previous two. Relaxing independence is one way to break the iid assumption and is the situation we consider. It can be done in several ways, we will discuss just two.

One of the ways which have been employed in the CTRW formalism is to take a power-law pdf of waiting times $p(\tau)$ $\sim \tau^{-(1+\alpha H)}$ [45]. This became known as the fractional time process (see also [25]). The waiting times themselves are still iid, but their infinite mean is assumed to be a consequence of dependence due to microscale physics. The kinetic equation that corresponds to the ftp $[25,46,47]$ can be seen in the fourth column of the eighth row in Table I. We may define a temporal exponent by $d^{\prime}=\alpha H$. The fractional derivative in time, of order $\alpha H=d^{\prime}$, corresponds physically to the power law in waiting times. The prime indicates that this exponent is not identical to the memory parameter $d$ in the case of $\mathrm{fBm}$ or fractional autoregressive integrated moving average (FARIMA) processes [24]. $d^{\prime}$ runs from 0 to 1 and is, for example, the same as the temporal exponent defined by Brockmann et al. [12] (their " $\alpha$;" our $\alpha$ is their " $\beta$ "). In all the following cases $D$ is no longer the Brownian diffusion constant but simply ensures dimensional correctness in a given equation. Note that we do not include the term describing the power-law decay of the initial value here or in subsequent CTRW equations (it is retained and discussed in [46]; see their Eq. 40).

Another way to relax independence is to introduce global long-range dependence, as pioneered by Mandelbrot and Van Ness [21]. They used a self-affine process with a memory kernel, originally due to Kolmogorov [17] and called by them "fractional Brownian motion." Contradictory statements exist in the physics literature concerning the equivalent kinetic equation for $\mathrm{fBm}$ corresponding to that for ftp. It has sometimes been asserted $[1,23]$ that the equations are the same, while ftp has sometimes been labeled "fBm" (cf. the supplementary material in [12]). However the solution of the equation for ftp is now known to be non-Gaussian $[5,46]$. Conversely the pdf of $\mathrm{fBm}$ is by definition $[48,49]$ Gaussian but with a variance which "stretches" with time as $t^{2 H}$. The correct kinetic equation for fBm must thus [25] be local in time. It is shown in row 8, column 3 of Table I. Given, to our knowledge, first by Wang and Lung [30], it can be seen by trial solution to have a solution of the required form.

The difference between ftp and $\mathrm{fBm}$ is striking, in that although both include temporal correlations, the kinetic equation for the ftp is nonlocal in time, while that for $\mathrm{fBm}$ is local. This distinction disappears when we go to a Langevin description, where both processes explicitly require fractional derivatives [25]. We are grateful to our referee for pointing out that the kinetic equation for $\mathrm{fBm}$ also corresponds to a transformation of time to $t^{2 H}$ in the ordinary diffusion equation for $\mathrm{BWBm}$, which we may contrast with the fractional derivative in time in the kinetic equation for ftp. We remark that if one rescales BWBm with time, the resulting increments would not be stationary, whereas fBm with the same kinetic equation has stationary increments. This illustrates the point that $\mathrm{fBm}$ shares its kinetic equation with several other stochastic processes and so a full specification of the process thus requires more than the kinetic equation.

\section{Combining memory with infinite variance: "Ambivalence" versus lfsm \\ 1. “Ambivalent processes" and the fully fractional continuous-time random walk}

Questions similar to those in Sec. II C have been asked in the physics literature about the natural generalization of the fractional time process to allow for both Lévy distributions of jump lengths as well as power-law-distributed waiting times. The resulting fully fractional kinetic equation is fractional in both space and time,

$$
\frac{\partial^{\alpha H}}{\partial t^{\alpha H}} p_{a p}(x, t)=D \frac{\partial^{\alpha}}{\partial|x|^{\alpha}} p_{a p}(x, t),
$$

and was used by Brockmann et al. [12] to exemplify the "ambivalent process." Analogously as with the ftp, the solution for this process is known [50] not to be a Lévy-stable (or stretched Lévy-stable) distribution but rather a convolution of such distributions.

\section{Self-similar stable alternative to the ap: Linear fractional stable motion}

Analogously as with the generalization of the ftp to the ambivalent process, there are several $H$-self-similar Levy symmetric $\alpha$-stable processes, enumerated in [51]. We here consider the simplest one, lfsm, which generalizes linear fractional Brownian motion to the infinite-variance case. We emphasize that lfsm is, for example, not the fractional Levy motion referred to by [52]. We can describe lfsm through a stochastic integral:

$$
S_{H}\left(t \mid \alpha, b_{1}, b_{2}\right)=\int_{-\infty}^{\infty} K_{H, \alpha}(t-s) Z_{\alpha}(d s)
$$

where the memory kernel $K_{H, \alpha}$ is given by 


$$
\begin{aligned}
K_{H, \alpha}= & b_{1}\left[(t-s)_{+}^{H-1 / \alpha}-(-s)_{+}^{H-1 / \alpha}\right] \\
& +b_{2}\left[(t-s)_{-}^{H-1 / \alpha}-(-s)_{-}^{H-1 / \alpha}\right] .
\end{aligned}
$$

Burnecki et al. [53] showed how mixed linear fractional stable motion can be obtained from $Z_{\alpha}(t)$ using the Lamperti transformation $[14,54]$, a more general result which enables any self-similar process to be obtained from its corresponding stationary stochastic process. We are concerned here, however, simply with obtaining the kinetic equation. This can be found by direct differentiation of the characteristic function with respect to time (cf. [55]).

As with the simpler stable processes the pdf $p_{\text {lfsm }}$ of lfsm can be expressed via the Fourier transform of the characteristic function (e.g., $[13,56]$ ),

$$
p_{\mathrm{lfsm}}(x)=\frac{1}{2 \pi} \int e^{-i k x} \exp \left(-\bar{\sigma}|k|^{\alpha} t^{\alpha H}\right) d k .
$$

We see that the characteristic function $\hat{p}(k)=\exp \left(-\bar{\sigma}|k|^{\alpha} t^{\alpha H}\right)$ generalizes the oLm case. Because $\alpha$ is no longer equal to $1 / H$ the effective width parameter now grows like $t^{\alpha H}$. The characteristic function has the correct $\mathrm{fBm}$ limit because when $\alpha=2$, we see for $\mathrm{fBm}$ at any given $t$ that it is a Gaussian with width growing as $t^{2 H}$. We can also see [28] that lfsm is a general stable self-affine process by taking $k^{\prime}=k \tau^{H}$, which gives $p_{\text {lfsm }}(x)=t^{-H} \phi_{\alpha}\left(x / t^{H}\right)$, a stable distribution of in$\operatorname{dex} \alpha$ and a prefactor ensuring $H$-self-similarity in time.

Direct differentiation of this pdf gives

$$
\frac{\partial}{\partial t} p_{\mathrm{lfsm}}=-\alpha H \frac{\bar{\sigma}}{2 \pi} t^{\alpha H-1} \int_{-\infty}^{\infty} e^{-i k x}|k|^{\alpha} \exp \left(-\bar{\sigma}|k|^{\alpha} t^{\alpha H}\right),
$$

which can be recognized as

$$
\frac{\partial}{\partial t} p_{\mathrm{lfsm}}=\alpha H t^{\alpha H-1} D \frac{\partial^{\alpha}}{\partial x^{\alpha}} p_{\mathrm{lfsm}}
$$

using the definition of the Riesz derivative in Sec. II B. Surprisingly the kinetic equation of lfsm seems to not have been given explicitly before in either the physics or mathematics literature. Krishnamurthy et al. [28] quoted an equation of motion for integrated activity in lfsm. This has a more complicated structure, presumably due to additional memory effects arising from the integration process.

We note that $\alpha H t^{\alpha H-1}=\partial_{t} t^{\alpha H}$. This factor arises because Eq. (8) could also be obtained from space fractional diffusion equation (2) by a simple transformation of the time variable: $t$ is replaced by $t^{\alpha H}$. The appropriate limits may be easily checked; in particular $\alpha=2$ gives the kinetic equation of $\mathrm{fBm}$.

We also remark that lfsm should be a special case of the nonlinear shot noise process studied by Eliazar and Klafter [57], which may allow further generalization of the kinetic equation we have presented.

\section{Ifsm AS A MODEL OF INTERMITTENT BURSTS}

Intermittency is a frequently observed property in complex systems, and can be studied within several paradigms. One such, of continuing interest, has been Bak et al.'s SOC, a key postulate of which is that slowly driven, interactiondominated, thresholded dynamical systems will establish long-ranged correlations via "avalanches" of spatiotemporal activity. The avalanches are found to obey power laws in size and duration. In consequence, many papers have sought to measure "bursts" of activity in natural time series. This has most typically been done by means of a fixed threshold. The duration $\tau$ and size $s$ of the bursts are then defined as the interval between the $i$ th upward crossing time $\left(t_{i}\right)$ and the next downward crossing time $\left(t_{i+1}\right)$ of the threshold, and the integrated area above the threshold between these times, respectively.

The search for SOC in the magnetosphere and solar wind has used this approach among others (e.g., [22,34]), while burst exponents have also been calculated in MHD turbulence models [35-37]. The similarity of observed burst size and duration distributions in solar wind and magnetospheric quantities to those from models of turbulence and SOC led Freeman et al. [34], Watkins [22], and Chapman et al. [58] to conjecture that, at least qualitatively, such behavior might simply be an artifact of a self-similar (or multifractal) time series, rather than unique to a given mechanism. In particular this was in distinction to the idea that one could use the presence or absence of power laws in waiting times defined similarly as the above to distinguish between SOC and turbulence. One of the present authors thus elsewhere [22] advocated the testing of avalanche diagnostics using controllable self-similar models. Similar points were by Carbone et al. [38] for fBm and Bartolozzi [39] for the multifractal random walk.

In this section we thus present a preliminary investigation of the ability of lfsm to qualitatively mimic SOC signatures in data. The kinetic equation we have derived is not unique to lfsm and is insufficient to specify all its properties, so in what follows we have used a numerical simulation of the process $S_{H}$ using the algorithm of Stoev and Taqqu [41] and analytic arguments based on those of Kearney and Majumdar [40] to predict the scaling of the tail of the pdf of burst size $s$ and duration $\tau$ in lfsm for large $s, \tau$. Carbone et al. [38] gave a similar argument to ours, but unlike us restricted its application to the $\mathrm{fBm}$ case. Rather than estimate the exponents from plots of the numerically simulated empirical pdf or complementary cumulative distribution function (cdf), we have elected to use maximum-likelihood estimation, as implemented in the algorithms of Clauset et al. [59]. Detailed comparison with measured experimental exponents is not attempted at this stage and will be the subject of future work.

Dealing first with durations, we make use of the fact that for a fractal curve of self-similarity exponent $H$ and dimension $D=2-H$, the points $\left\{t_{i}\right\}$ have dimension $1-H$. In consequence the probability of crossings over a time interval $\tau$ goes as $\tau^{1-H}$, giving an interevent probability scaling like $\tau^{-(1-H)}$. The pdf for interevent intervals in the isoset thus scales as

$$
p(\tau) \sim \tau^{\beta}
$$

where 


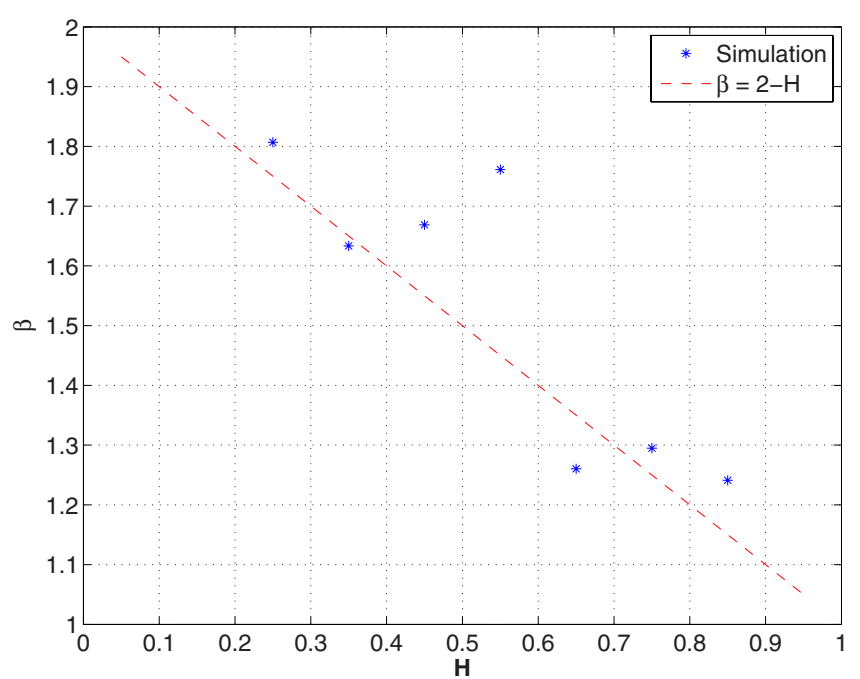

FIG. 1. (Color online) Same as Fig. 3, dependence of duration exponent $\beta$ on $H$, but one trial only.

$$
\beta=2-H
$$

giving the same exponent of $3 / 2$ as for the first-passage distribution in the Brownian case. For symmetric processes this scaling is retained by the subset of the isoset that corresponds to burst "durations" (e.g., [34,38]). We expect this to be independent of the detailed nature of the model and so should, in particular, also apply to lfsm.

To establish the behavior of burst "sizes" we note first that Kearney and Majumdar [40] considered the zero-drift BWBm case. Rather than their full analytic treatment, we recap their heuristic argument for a burst size (area) $A$ defined using the first-passage time $t_{f}$. This may then be adapted to burst sizes defined using isosets, and thence to lfsm. Kearney and Majumdar [40] first noted that for BWBm the instantaneous value of the random walk $y(t) \sim t^{1 / 2}$ for large $t$. Then, defining $A$ as

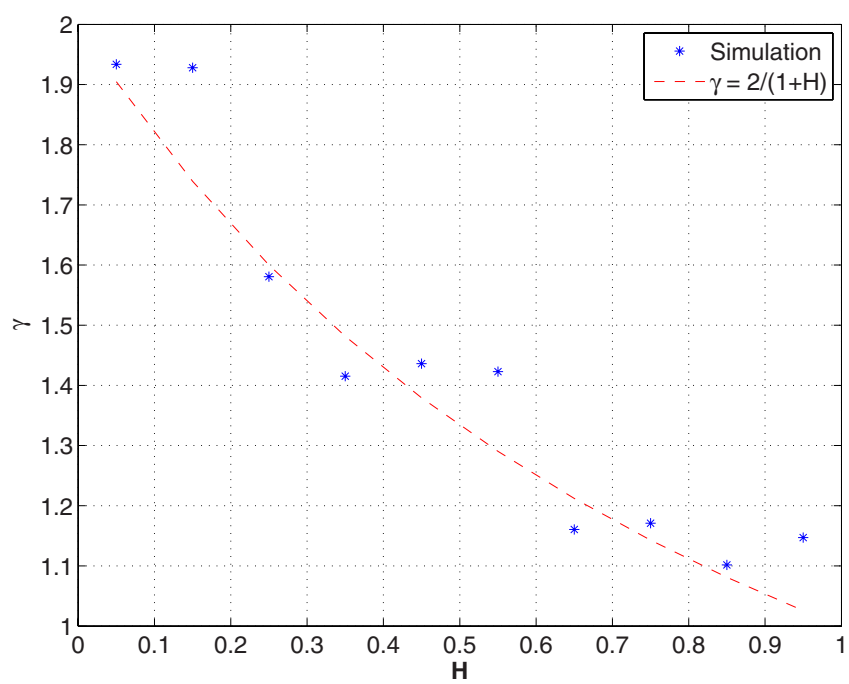

FIG. 2. (Color online) Same as Fig. 4, dependence of size exponent $\gamma$ on $H$, again one trial only.

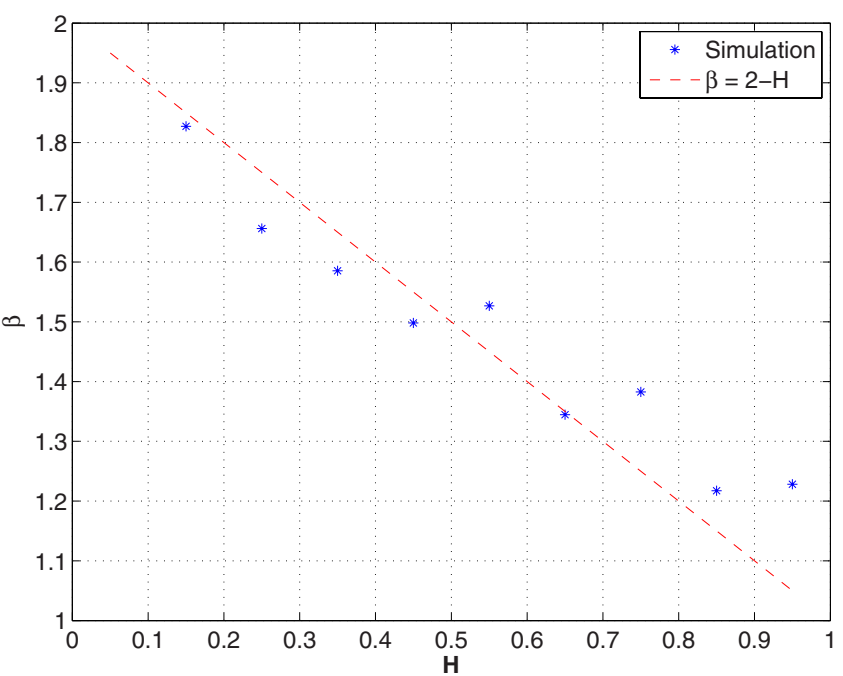

FIG. 3. (Color online) Dependence of exponent $\beta$ for $\operatorname{pdf} p(\tau)$ of a burst of duration $\tau$ on $H$ for simulated lfsm in the fBm, $\alpha=2$ limit. The average of seven trials was taken.

$$
A=\int_{t_{i}}^{t_{f}} y\left(t^{\prime}\right) d t^{\prime},
$$

the integration implies that large $A$ scales as $t_{f}^{3 / 2}$. Simple inversion of this expression implies that $t_{f}$ must scale as $t_{f}$ $\sim A^{2 / 3}$. We independently have the standard result for firstpassage time for BWBm: $P\left(t_{f}\right) \sim t_{f}^{-3 / 2}$. To get $P\left(t_{f}\right)$ as a function of $A$, i.e., $P\left(t_{f}(A)\right)$ one needs to insert the expression for $t_{f}$ as a function of $A$ in above equation, and in addition will need a Jacobian. After these manipulations $P(A) \sim A^{-4 / 3}[40]$.

In the zero-drift but non-Brownian case we will still argue that $y(t) \sim t^{H}$ for large $t$. As our application uses the abovementioned isoset-based burst size $s$ rather than those based on first-passage times, we define $s$ as

$$
s=\int_{t_{i}}^{t_{i+1}} y\left(t^{\prime}\right) d t^{\prime} .
$$

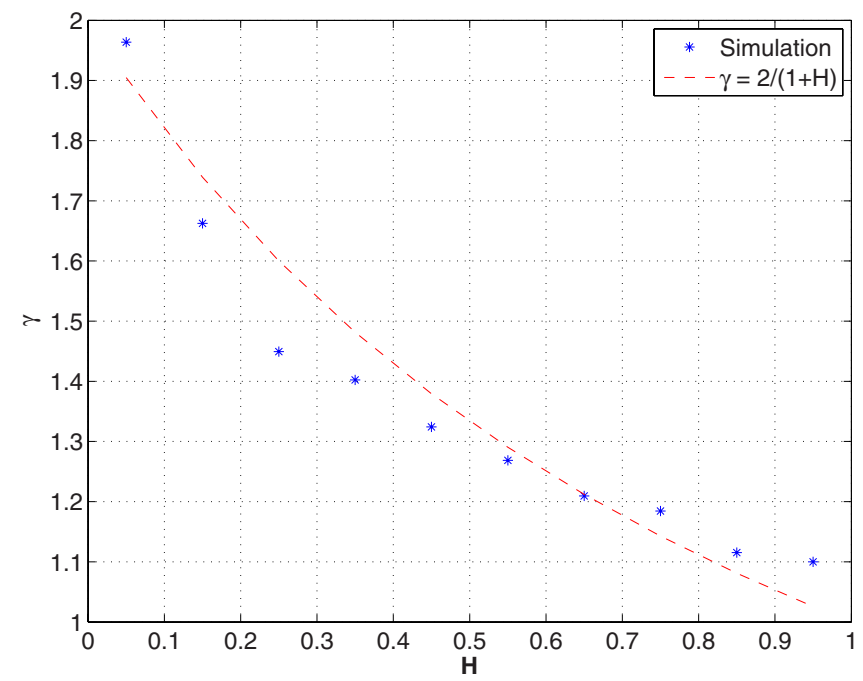

FIG. 4. (Color online) Dependence of exponent $\gamma$ for the pdf $p(s)$ of a burst of size $s$ on $H$ for simulated lfsm in fBm limit. Again an average of seven trials was taken. 


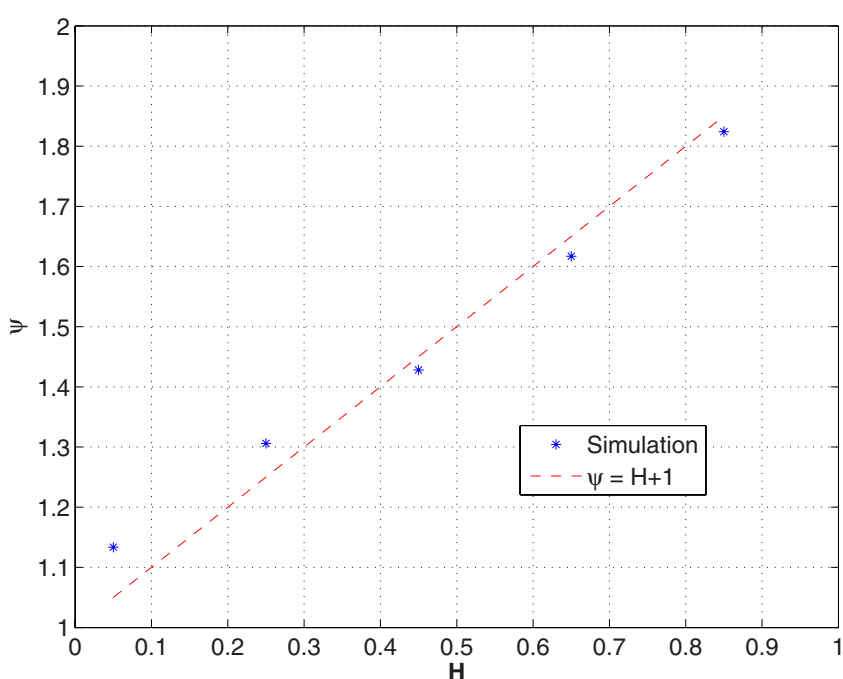

FIG. 5. (Color online) Dependence of exponent $\psi$ on $H$ in $\mathrm{fBm}$ case. $\psi$ captures growth of burst size $s$ with duration $\tau$.

The rest of the argument goes as before but using Eq. (9). We find

$$
P(s) \sim s^{\gamma}
$$

where

$$
\gamma=-2 /(1+H)
$$

which we can check in the Brownian case where $H=1 / 2$ to retrieve $P(s) \sim s^{-4 / 3}$.

The same exponents, $\beta$ and $\gamma$, but defining the bursts using a detrended fluctuation analysis (DFA)-like moving average rather than a fixed threshold, were earlier investigated, for the fBm case only, by Carbone et al. [38]. We have used the same notation, and the format of our figures for the $\mathrm{fBm}$ and lfsm cases has been chosen to allow comparison with theirs. They found the same dependences of $\beta$ and $\gamma$ on $H$ that we have in Eqs. (10) and (14) above, which is intuitively

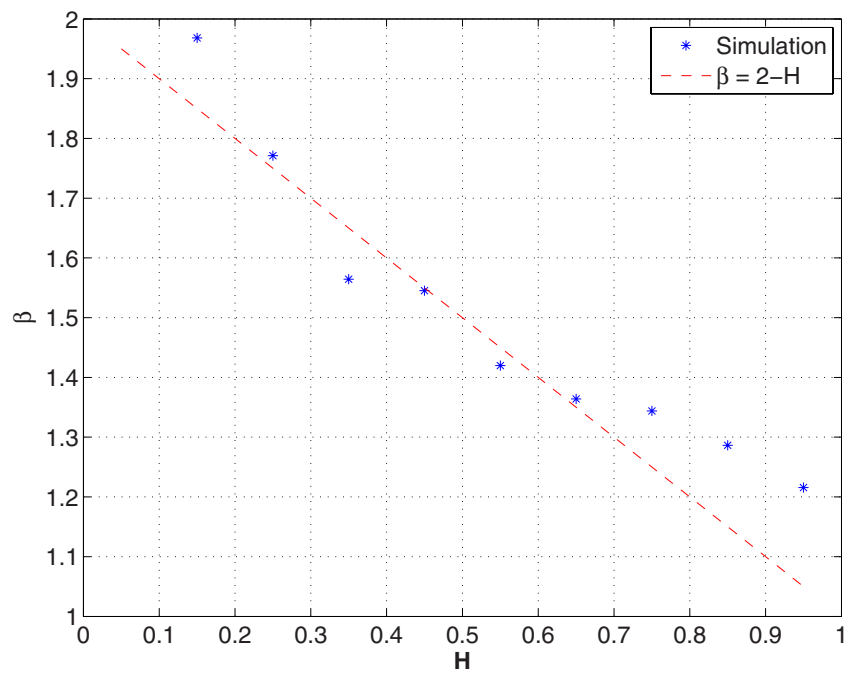

FIG. 6. (Color online) Same as Fig. 3 (burst duration exponent $\beta$ vs $H$; seven trials), but for $\alpha=1.8$.

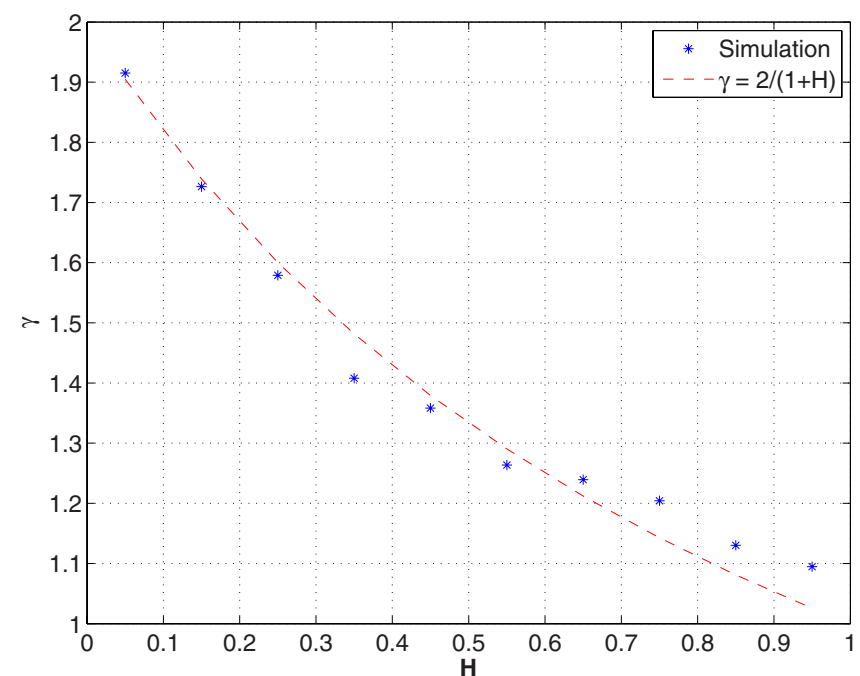

FIG. 7. (Color online) Same as Fig. 4 (burst size exponent $\gamma$ vs $H$; seven trials), but for $\alpha=1.8$.

reasonable on hindsight because the choice of fixed or running threshold should not change the asymptotic scaling behavior. For a fixed threshold the burst size and duration exponents for fBm were also very recently presented in [42].

A time series of 26768 points of lfsm was simulated for each $[H, \alpha]$ pair, from which the burst size data set was obtained. We then used MLE to estimate the exponents, on the assumption of a power-law pdf. Numerics confirm that using our fixed threshold definition the expressions obtained by earlier authors for $\beta$ and $\gamma$ describe fBm reasonably well, although the scatter, from a single trial in the case of each value of $\beta$ and $\gamma$ shown in Figs. 1 and 2, seems relatively high. We reduced the scatter in Figs. 3 and 4 by plotting the average of the exponents over a small number (here 7) of trials. The assumption that burst size $s$ grows with duration $\tau$ used in the heuristic derivation above can be seen to be reasonable for the fixed threshold, fBm case in Fig. 5.

Perhaps more surprisingly the expressions also seem to hold reasonably well when the stability exponent is reduced,

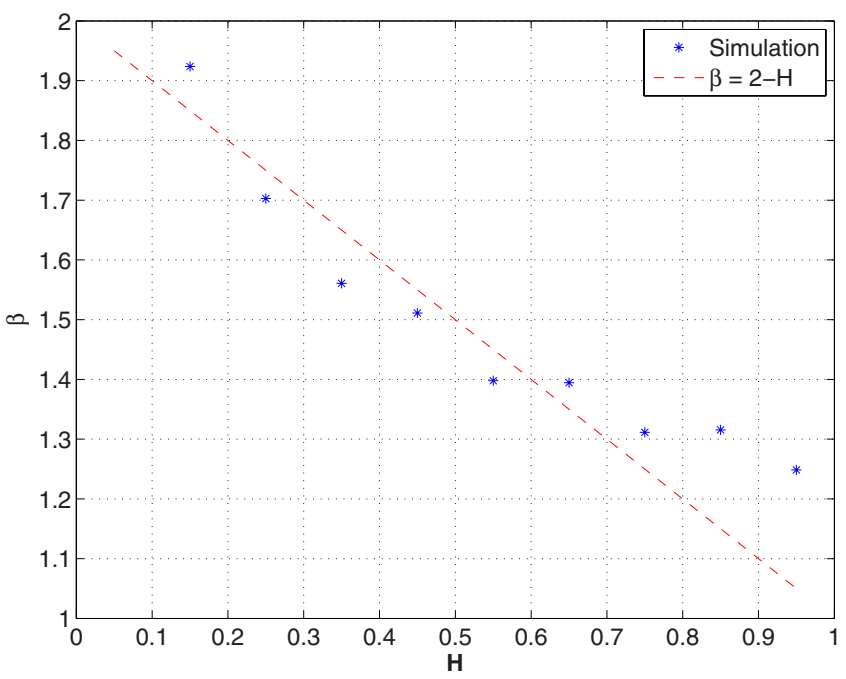

FIG. 8. (Color online) Same as Fig. 3 (burst duration exponent $\beta$ vs $H$; seven trials), but for $\alpha=1.6$. 


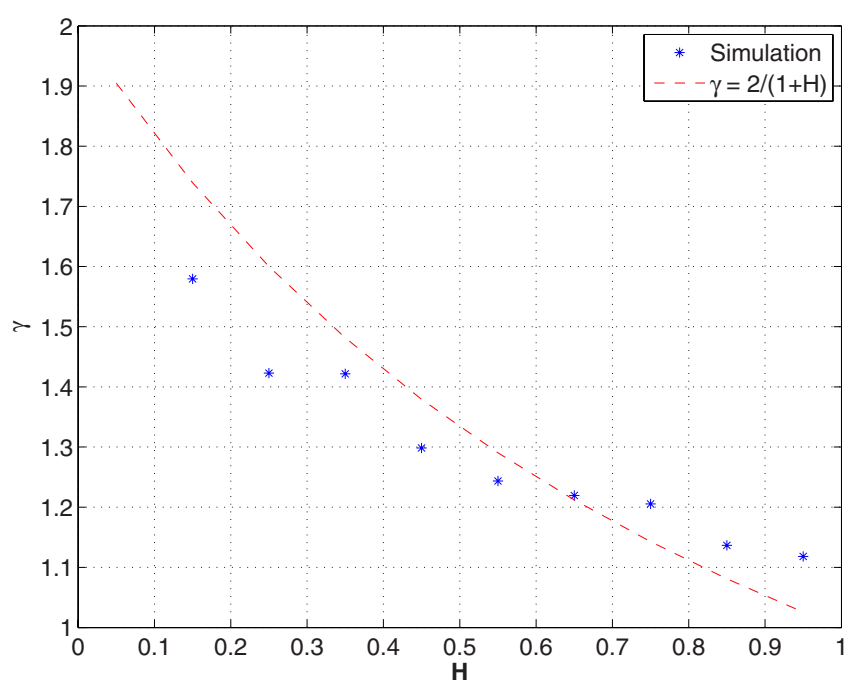

FIG. 9. (Color online) Same as Fig. 4 (burst size exponent $\gamma$ vs $H$; seven trials), but for $\alpha=1.6$.

first to 1.8 (Figs. 6 and 7) and then to 1.6 (Figs. 8 and 9). Again we note that these are averages of seven trials in each case. By the $\alpha=1$ case presented in Figs. 10 and 11, however, the expressions can clearly be seen to fail. In this parameter regime, for any given $H$, they are seen to consistently underestimate both the burst exponents. It has been suggested to us that this could be because $y^{H}$ ceases to be a good estimate of characteristic displacement when the increments of the walk are very heavy tailed [60], but we have so far been unable to find a suitable alternative expression. We also note that if the burst sizes and durations are not asymptotically distributed as power laws, then the departure from pure scaling must be incorporated into the MLE, or exponent estimates will be biased. Such MLEs exist [59], but we have left investigation of these issues to future work.

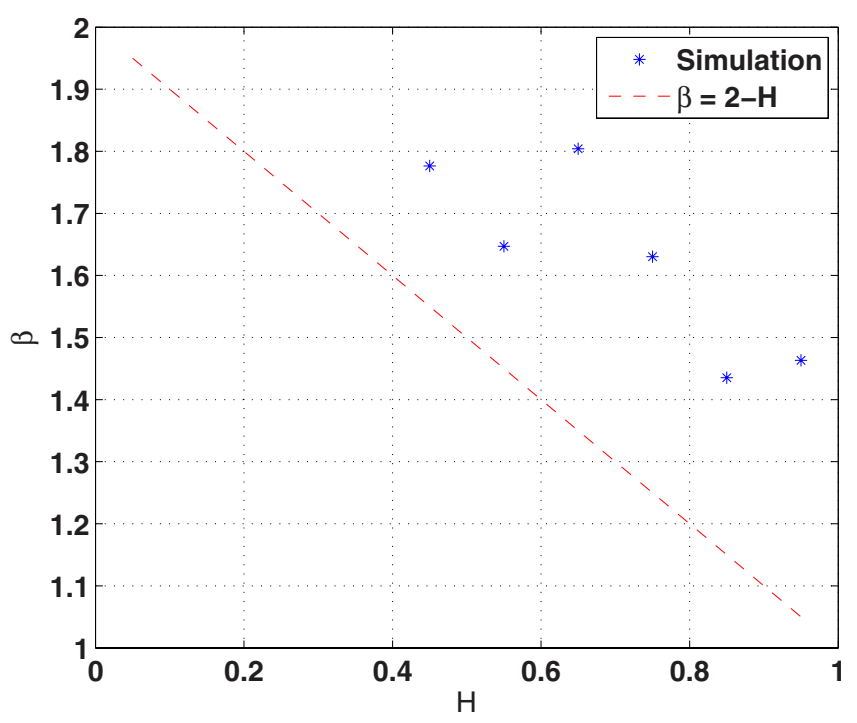

FIG. 10. (Color online) Same as Fig. 3 (burst duration exponent $\beta$ vs $H$; seven trials), but for $\alpha=1$.

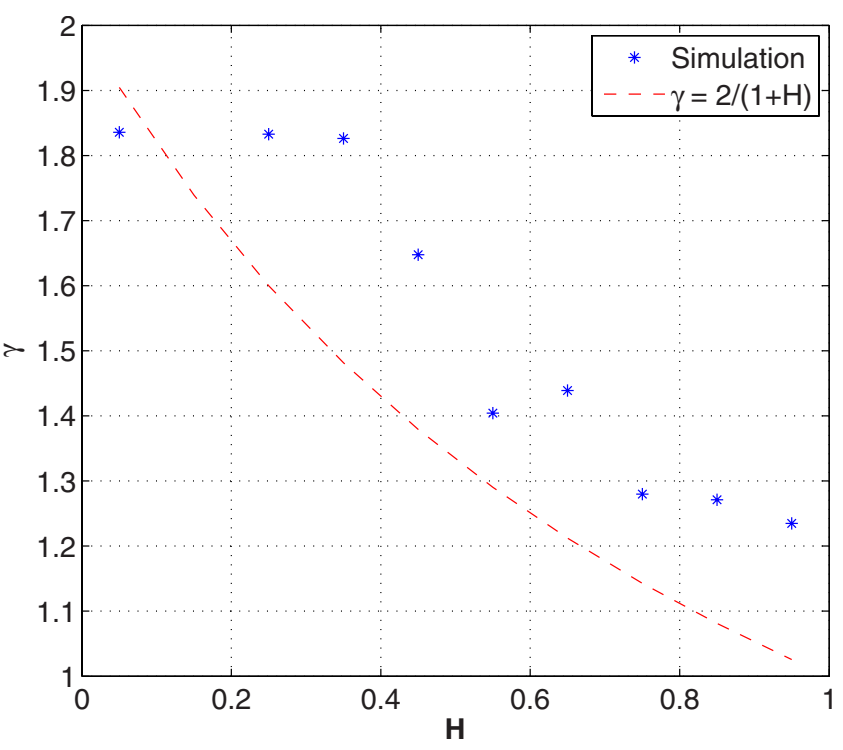

FIG. 11. (Color online) Same as Fig. 4 (burst size exponent $\gamma$ vs $H$; seven trials), but for $\alpha=1$.

\section{CONCLUSIONS}

In this paper we studied the question of whether one would expect the same equation to describe a time series as an anomalous diffusive process. A codification of diffusionlike equations showed that a kinetic equation was "missing" from the literature, the one corresponding to lfsm. We gave a simple derivation for it by direct differentiation of the wellknown characteristic function of lfsm. We then made a preliminary exploration of how lfsm could model the burst sizes and durations previously measured on magnetospheric and solar wind time series. We made simple scaling arguments building on a result of Kearney and Majumdar [40] to show how lfsm could be one candidate explanation for such "apparent SOC" behavior and made preliminary comparison with numerics. These arguments fail when the tails of the pdf of increments become very heavy, and further work is needed on this topic.

In future we also plan to consider other stochastic processes, both FARIMA (cf. [24]), nonlinear shot noises, and multifractals, to allow generalization of the above initial investigations into burst size and duration. The prevalence of natural processes showing heavy tails and/or long-ranged persistence suggests a relevance well beyond our initial area of application in space physics.

\section{ACKNOWLEDGMENTS}

We thank in particular our anonymous referee for numerous detailed and constructive suggestions, and Alex Weron, Krzysztof Burnecki, and Marcin Magdziarz for many helpful comments on earlier versions of the paper. We appreciate the public provision by Stilian Stoev and Aaron Clauset of the algorithms and codes from the papers [41,59]. N.W.W. is also grateful to Mikko Alava, Robin Ball, Tom Chang, Aleksei Chechkin, Joern Davidsen, Mervyn Freeman, Bogdan Hnat, Mike Kearney, Khurom Kiyani, Yossi Klafter, Vassili 
Kolokoltsev, Eric Lutz, Satya Majumdar, Martin Rypdal, Cosma Shalizi, Vadim Uritsky, Dimitri Vyushin, and Lev Zelenyi for valuable interactions. N.W.W. acknowledges the stimulating environments of the Newton Institute program PDS03 and the KITP program "The Physics of Climate
Change." Research was carried out in part at Oak Ridge National Laboratory, managed by UT-Battelle, LLC, for U.S. DOE under Contract No. DE-AC05-00OR22725. This research was supported in part by the EPSRC-GB, STFC, and NSF under Grant No. NSF PHY05-51164.
[1] G. M. Zaslavsky, Hamiltonian Chaos and Fractional Dynamics (Oxford University Press, Oxford, 2004).

[2] R. Balescu, Aspects of Anomalous Transport in Plasmas (Institute of Physics, Bristol, 2005).

[3] Anomalous Transport, edited by R. Klages, G. Radons, and I. M. Sokolov (Wiley, Weinheim, 2008).

[4] J. Klafter, M. F. Shlesinger, and G. Zumofen, Phys. Today 49(2), 33 (1996).

[5] E. Scalas, R. Gorenflo, and F. Mainardi, Phys. Rev. E 69, 011107 (2004).

[6] A. Mura, M. S. Taqqu, and F. Mainardi, Physica A 387, 5033 (2008).

[7] A. V. Milovanov and L. M. Zelenyi, Phys. Rev. E 64, 052101 (2001).

[8] R. Balescu, Phys. Rev. E 51, 4807 (1995).

[9] B. A. Carreras, V. E. Lynch, and G. M. Zaslavsky, Phys. Plasmas 8, 5096 (2001).

[10] D. del-Castillo-Negrete, B. A. Carreras, and V. E. Lynch, Phys. Rev. Lett. 94, 065003 (2005).

[11] R. Sánchez, B. Ph. van Milligen, and B. A. Carreras, Phys. Plasmas 12, 056105 (2005).

[12] D. Brockmann, L. Hufnagel, and T. Geisel, Nature (London) 439, 462 (2006).

[13] G. Samorodnitsky and M. S. Taqqu, Stable Non-Gaussian Random Processes (Chapman and Hall, New York, 1994).

[14] P. Embrechts and M. Maejima, Selfsimilar Processes (Princeton University Press, Princeton, 2002).

[15] T. Sottinen, Ph.D. thesis, University of Helsinki, 2003 (unpublished).

[16] R. N. Mantegna and H. E. Stanley, An Introduction to Econophysics (Cambridge University Press, Cambridge, England, 2000).

[17] A. N. Kolmogorov, Foundations of the Theory of Probability (Chelsea, New York, 1956); translation from German of Grundbegriffe der Wahrscheinlichkeitsrechnung (Julius Springer, Berlin, 1933).

[18] P. Billingsley, Probability and Measure (Wiley, Chichester, 1979).

[19] B. B. Mandelbrot, J. Business 36, 394 (1963).

[20] B. B. Mandelbrot, Gaussian Self-Affinity and Fractals: Globality, the Earth, 1/f Noise, and R/S (Springer, Berlin, 2002).

[21] B. B. Mandelbrot and J. W. Van Ness, SIAM Rev. 10, 422 (1968).

[22] N. W. Watkins, Nonlinear Processes Geophys. 9, 389 (2002).

[23] N. W. Watkins, D. Credgington, B. Hnat, M. P. Freeman, S. C. Chapman, and J. Greenhough, Space Sci. Rev. 121, 271 (2005).

[24] K. Burnecki, J. Klafter, M. Magdziarz, and A. Weron, Physica A 387, 1077 (2008).

[25] E. Lutz, Phys. Rev. E 64, 051106 (2001).
[26] G. M. Zaslavsky, P. N. Guzdar, M. Edelman, M. I. Sitnov, and A. S. Sharma, Physica A 373, 11 (2007).

[27] G. M. Zaslavsky, P. N. Guzdar, M. Edelman, M. I. Sitnov, and A. S. Sharma, Commun. Nonlinear Sci. Numer. Simul. 13, 314 (2008).

[28] A. Krishnamurthy, A. Tanguy, P. Abry, and S. Roux, Europhys. Lett. 51, 1 (2000).

[29] N. W. Watkins, D. Credgington, R. Sanchez, and S. C. Chapman, e-print arXiv:0803.2833.

[30] K. G. Wang and C. W. Lung, Phys. Lett. A 151, 119 (1990).

[31] I. Calvo, R. Sanchez, and B. A. Carreras, J. Phys. A: Math Theor. 42, 055003 (2009).

[32] S. Mercik, K. Weron, K. Burnecki, and A. Weron, Acta Phys. Pol. B 34, 3773 (2003).

[33] D. Sornette, Critical Phenomena in Natural Sciences, 2nd ed. (Springer, Berlin, Germany, 2004).

[34] M. P. Freeman, N. W. Watkins, and D. J. Riley, Phys. Rev. E 62, 8794 (2000).

[35] P. Dmitruk and D. O. Gomez, Astrophys. J. 484, L83 (1997).

[36] S. Galtier and A. Pouquet, Sol. Phys. 179, 141 (1998).

[37] N. W. Watkins, S. Oughton, and M. P. Freeman, Planet. Space Sci. 49, 1233 (2001).

[38] A. Carbone, G. Castelli, and H. E. Stanley, Phys. Rev. E 69, 026105 (2004); A. Carbone and H. E. Stanley, Physica A 340, 544 (2004). Note that the analytic derivation in these papers of an expression for $\gamma$ needs a minor correction. Referring to the PRE, the left-hand side of Eq. 15 is not equal to the right-hand side when the definitions of $\beta$ and $\psi_{\text {s }}$ are substituted; it should instead read $\gamma=\left(\beta-1+\psi_{s}\right) / \psi_{s}$.

[39] M. Bartolozzi, Eur. Phys. J. B 57, 337 (2007).

[40] M. J. Kearney and S. N. Majumdar, J. Phys. A 38, 4097 (2005).

[41] S. Stoev and M. Taqqu, Fractals 12, 95 (2004).

[42] M. Rypdal and K. Rypdal, Phys. Rev. E 78, 051127 (2008).

[43] P. Lévy, Theorie de l'Addition des Variables Aleatoires (Gauthier-Villars, Paris, 1937).

[44] M. M. Meerschaert and H. P. Scheffler, Limit Distributions for Sums of Independent Random Vectors: Heavy Tails in Theory and Practice (Wiley, Hoboken, NJ, 2001).

[45] K. Lindenberg and B. J. West, J. Stat. Phys. 42, 201 (1986).

[46] R. Metzler and J. Klafter, Phys. Rep. 339, 1 (2000).

[47] V. Balakrishnan, Physica A 132, 569 (1985).

[48] J. McCauley, Dynamics of Markets: Econophysics and Finance (Cambridge University Press, Cambridge, England, 2004).

[49] B. B. Mandelbrot, The Fractal Geometry of Nature (Freeman, San Francisco, 1982).

[50] V. Kolokoltsov, V. Korolev, and V. Uchaikin, J. Math. Sci. (N.Y.) 105, 2569 (2001).

[51] A. Weron, K. Burnecki, S. Mercik, and K. Weron, Phys. Rev. E 
71, 016113 (2005).

[52] T. Huillet, J. Phys. A 32, 7225 (1999).

[53] K. Burnecki, M. Maejima, and A. Weron, Yokohama Math. J. 44, 25 (1997)

[54] J. Lamperti, Trans. Am. Math. Soc. 104, 62 (1962).

[55] W. Paul and J. Baschnagel, Stochastic Processes: From Physics to Finance (Springer, Berlin, 1999).

[56] N. Laskin, I. Lambadaris, F. C. Harmantzis, and M. Devetsiki- otis, Comput. Netw. 40, 363 (2002).

[57] I. Eliazar and J. Klafter, Physica A 360, 227 (2006).

[58] S. C. Chapman, R. O. Dendy, and N. W. Watkins, Plasma Phys. Controlled Fusion 46, B157 (2004).

[59] A. Clauset, C. R. Shalizi, and M. E. J. Newman, e-print arXiv:0706.1062.

[60] S. Majumdar (private communication). 